\title{
Synthesis, characterization, photocatalytic and reusability studies of capped ZnS nanoparticles
}

\author{
JAGDEEP KAUR, MANOJ SHARMA ${ }^{\dagger}$ and O P PANDEY* \\ School of Physics and Materials Science, Thapar University, Patiala 147 004, India \\ ${ }^{\dagger}$ Department of Nanotechnology, Sri Guru Granth Sahib World University, Fatehgarh Sahib 140 406, India
}

MS received 21 June 2013; revised 15 July 2013

\begin{abstract}
This paper presents results of a study on the structural and morphological properties of 2-mercaptoethanol (2-ME) capped ZnS nanoparticles (NPs). The photocatalytic and reusability study of the synthesized NPs to degrade dyes was also done. ZnS semiconductor NPs were synthesized via chemical precipitation route using 2-ME as a stabilizing agent. The as-prepared NPs were characterized by $\mathrm{X}$-ray diffraction (XRD) technique to confirm the nanometer sized particle formation. Morphological features of capped ZnS NPs were determined by transmission electron microscopy (TEM). Dynamic light scattering (DLS) technique was used to determine the hydrodynamic size of capped ZnS NPs. UV-Vis studies were done to determine the absorption edge and bandgap of the capped ZnS NPs. Fourier transform infrared spectroscopy (FT-IR) studies were done to confirm the presence of 2-ME on the surface of NPs. Photocatalytic studies of the as-prepared ZnS NPs were done by taking Ponceau S and crystal violet dyes as model pollutants. Their comparative degradation behaviour has been discussed. Reusability study of ZnS NPs was done to ensure its applicability as recycled catalyst in photocatalysis. The result showed photocatalytic enhancement of reused catalyst. Possible reason has been discussed in this work.
\end{abstract}

Keywords. ZnS nanoparticles; capping; dye degradation; reusability.

\section{Introduction}

Textile industry uses large volumes of water in wet processing operations resulting in generation of substantial quantities of wastewater which contain large amount of dyestuff. Although these dyes are an important part of textile industry but their discharge in water bodies is very complicated as these are highly toxic and have carcinogenic effects on human health (Chung 1983; Drinkwater 1990; Chung et al 1992). These dyes often have complex aromatic structure and do not degrade easily under natural conditions as these are highly photostable. The presence of very small amount of dye in water $(<1 \mathrm{ppm}$ for some dyes) is visible and undesirable (Banat et al 1996). Till now, many conventional methods (chemical, physical, biological) have been developed to overcome this problem (Robinson et al 2001). These methods have their own advantages and disadvantages. To overcome the disadvantages of these conventional methods, development of a cost effective method is required which can effectively degrade the dyes without forming any sludge or other toxic substances. Unlikely conventional methods of colour removal, semiconductor assisted photocatalytic degradation of dyes, which is an advanced oxidation

*Author for correspondence (oppandey@thapar.edu) process (AOP) has emerged as an important destructive technology that offers complete mineralization of most of the organic pollutants and thus there is no further requirement for secondary disposal methods (Hoffmann et al 1995; Sarkar et al 2013). In recent years, semiconductor nanostructures have been studied extensively to explore their size dependent optical properties and potential applications in various areas such as optical sensitization and photocatalysis (Kanemoto et al 1992; Olad et al 2012). It has been reported by various research groups that nanostructures exhibit superior photocatalytic activities due to the increased surface/volume ratio with enhanced redox potential as compared to their bulk counterpart. Among the various catalysts, ZnS nanoparticles have been proved as a better photocatalyst due to rapid generation of electronhole pairs with photoexcitation as it is a direct wide bandgap semiconductor material (Jiang et al 2009). Further, it possesses high negative reduction potential of excited electrons due to its higher conduction band position in an aqueous solution as compared to other extensively studied photocatalysts (Li et al 2011). Also, these NPs show good photocatalytic activity due to trapped holes arising from surface defects on the sulphides (Yanagida et al 1986). A good photocatalyst should retain its properties so that it can be reused in the dye degradation process. Another factor which plays an important role in degradation of dye is $\mathrm{pH}$ which determines the 
nature of surface charge of the catalyst to facilitate its adsorption on the surface of dye in solution. Taking into account the above important points, emphasis in the present work is to synthesize capped $\mathrm{ZnS}$ nanoparticles and to study their structural, optical, photocatalytic and reusability properties. In this context, an attempt was made to study the effect of $\mathrm{pH}$ (of both dye and catalyst solution) on degradation of cationic and anionic dyes.

The model of water pollutant selected in this study is Ponceau S (dye 1) (also known as acid red 112 or C.I. 27195) and crystal violet (dye 2) (also known as gentian violet, methyl violet 10B, hexamethyl pararosaniline chloride or pyoctanine). Dye 1 has molecular formula $\mathrm{C}_{22} \mathrm{H}_{12} \mathrm{~N}_{4} \mathrm{Na}_{4} \mathrm{O}_{13} \mathrm{~S}_{4}$. It is a sodium salt of a diazo dye that is mainly used in clinical laboratories as a proteinbinding dye for staining of blood serum proteins (Bannur et al 1999). It is also used in textile industry, leather and paper (Meena et al 2009; El-Desoky et al 2010). Till date, there are no results reported which directly emphasized on the toxic effects of this dye. However, its biotransformation products have toxic effects against aquatic organisms and suspicious of being carcinogenic for human beings (Chung 1983; Chung et al 1992). To the best of our knowledge, this dye has not been degraded yet by employing ZnS NPs as a photocatalyst. Dye 2 is a triarylmethane dye with molecular formula $\mathrm{C}_{25} \mathrm{~N}_{3} \mathrm{H}_{30} \mathrm{Cl}$. It is widely used in paper industry to dye paper. Although this dye has many applications in medical and non-medical area, but it is very toxic to aquatic organisms as it may cause long-term adverse effects in the aquatic environment. The exposure to this dye can have possible risk of irreversible effects on mankind and may even cause cancer (Drinkwater 1990). Molecular structure of both dyes are given in figure 1.

\section{Experimental}

\subsection{Materials and reagents}

Zinc acetate $\left(\mathrm{Zn}\left(\mathrm{CH}_{3} \mathrm{COO}\right)_{2}\right)$, 2-mercaptoethanol $\left(\mathrm{HSCH}_{2}\right.$ $\left.\mathrm{CH}_{2} \mathrm{OH}\right)$, sodium sulphide nanohydrate $\left(\mathrm{Na}_{2} \mathrm{~S} \cdot 9 \mathrm{H}_{2} \mathrm{O}\right)$, crystal violet $\left(\mathrm{C}_{25} \mathrm{~N}_{3} \mathrm{H}_{30} \mathrm{Cl}\right)$ and Ponceau $\mathrm{S}\left(\mathrm{C}_{22} \mathrm{H}_{12} \mathrm{~N}_{4}\right.$ $\mathrm{Na}_{4} \mathrm{O}_{13} \mathrm{~S}_{4}$ ) were purchased from Sigma Aldrich and were of high purity (99.99\%). All chemicals used in this work were of analytical grade and were used in as received condition.

\subsection{Apparatus for photocatalytic study}

Photocatalytic degradation experiment was conducted in dark using self-designed photochemical reactor at room temperature. The apparatus consisted of a cylindrical glass cell of $1 \mathrm{~L}$ capacity. A mercury lamp $(\lambda=300-400 \mathrm{~nm})$ was placed in a glass tube. The lamp and tube were then immersed in a water jacket to cool the system. The glass cylinder was filled with $10 \mathrm{mg} / \mathrm{L}$ of dye solution and $2.5 \mathrm{~g} / \mathrm{L}$ of catalyst. The resulting solution was stirred using magnetic stirrer for $30 \mathrm{~min}$ to ensure adsorption/ desorption equilibrium of the dye on the photocatalyst surface. After that, UV lamp was switched onto start the photocatalytic process. To determine the percentage degradation of dye, the samples were collected at regular intervals of time $(60 \mathrm{~min})$ and centrifuged to remove the catalyst that existed as undissolved particles in the samples.

Dyes 1 and 2 both absorb in visible region at around 519.19 and $572.41 \mathrm{~nm}$, respectively. Therefore, photometric analysis of samples before and after UV irradiation can be used to study their percentage degradation. The absorbance of supernatant was measured by a UVVis spectrophotometer Hitachi U-3900H (range 200$650 \mathrm{~nm}$ ) using a paired quartz cuvette.

\subsection{Adsorption studies}

To ensure whether adsorption of dye is taking place on the surface of catalyst or not, an experiment was conducted in dark. $10 \mathrm{mg} / \mathrm{L}$ of dye (dye 1) solution and $2.5 \mathrm{~g} / \mathrm{L}$ of catalyst were put in a glass cylinder and were stirred for $3 \mathrm{~h}$. A known amount of the above solution was taken out after regular intervals of time (60 min). Rest of the procedure followed has already been described in §2.2. A small decrease in the concentration of dye 1 as shown in figure 2 suggested that the dye molecules were able to adsorb on the surface of catalyst sufficiently.

\subsection{Experimental}

ZnS NPs were synthesized by chemical precipitation method by adding appropriate amount of zinc acetate
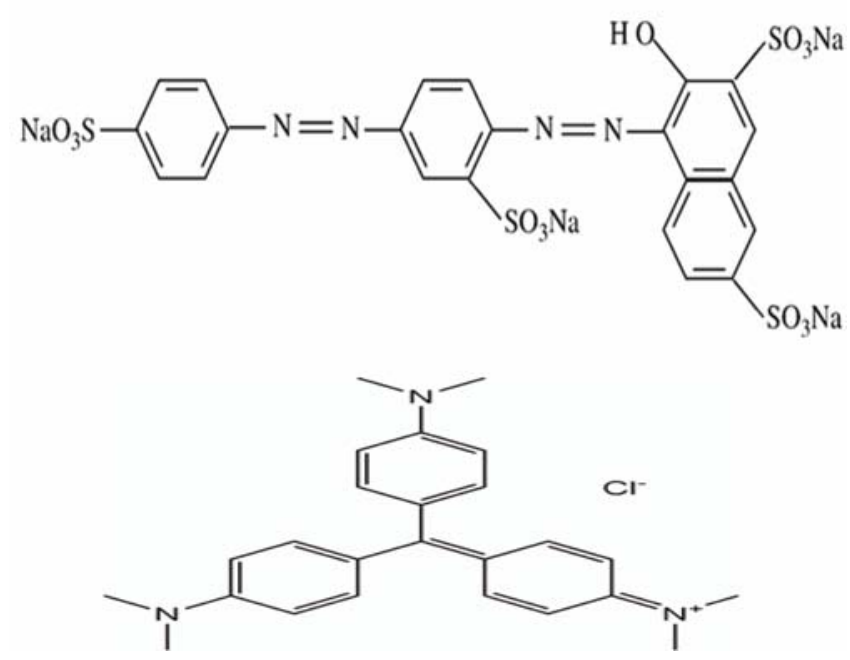

Figure 1. Molecular structure of (a) dye 1 and (b) dye 2 . 
solution and $S^{2-}$ as precipitating anion formed by decomposition of sodium sulphide nanohydrate $\left(\mathrm{Na}_{2} \mathrm{~S} \cdot 9 \mathrm{H}_{2} \mathrm{O}\right)$ (Sharma et al 2012). To prepare capped ZnS NPs, 0·25 M zinc acetate was dissolved in ultrapure water. The solution was stirred for 30 min using magnetic stirrer. 2-ME was used as capping agent to prevent agglomeration of NPs. 1\% solution of 2-ME was prepared in ultrapure water and stirred. To the stirred solution of zinc acetate, solution of capping agent was poured drop by drop. After $30 \mathrm{~min}$, solution of sodium sulphide was poured drop by drop. Very fine precipitates appeared soon after the addition of sodium sulphide, indicating the precipitation phenomenon occurring in the system. $\mathrm{pH}$ of the solution was monitored and it came out to be $5 \cdot 26$. After that, stirring was done for $3 \mathrm{~h}$. The entire experiment mentioned above was done at room temperature. The as-prepared solution was centrifuged at $10,000 \mathrm{rpm}$ and the precipitates were washed several times with distilled water to remove the last traces of adhered impurities. The washed particles were dried in an oven at $60^{\circ} \mathrm{C}$ for $24 \mathrm{~h}$ and then crushed to obtain a fine powder.

\subsection{Characterization techniques}

ZnS NPs were characterized by X-ray diffraction (XRD) technique using Panalytical's X'Pert Pro diffractometer with $\mathrm{CuK} \alpha$ radiation. Micrograph was recorded using the analytical transmission electron microscope (TEM, PHILIPS, Model-CM 200) having accelerating voltage of $300 \mathrm{kV}$. DLS studies were conducted using 90 Plus/BI-MAS (Brookhaven Instruments Corporation). Optical absorption spectra of ZnS NPs was recorded with a double beam UV-Vis spectrophotometer (Model: Hitachi U-3900H) in the range $190-650 \mathrm{~nm}$. FT-IR spectrum was recorded using Perkin Elmer-Spectrum RX-IFTIR.

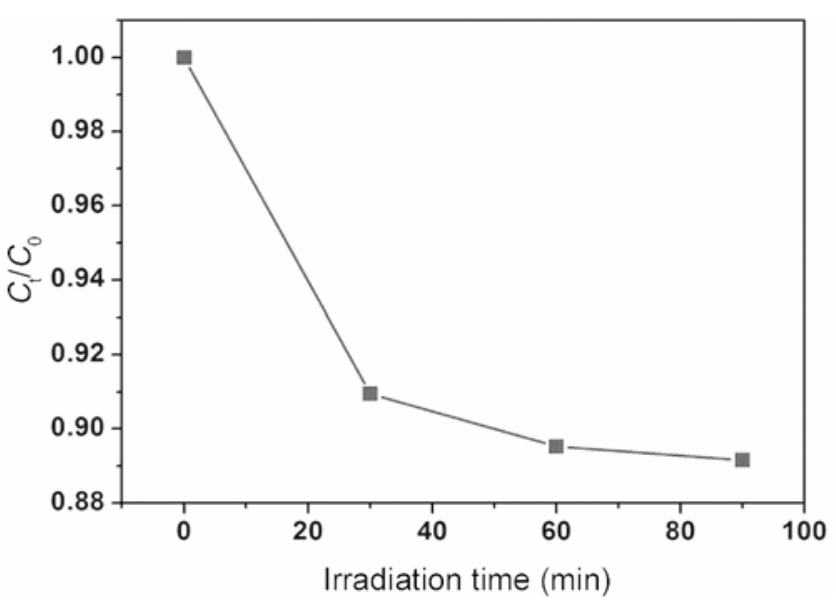

Figure 2. Variation in $C_{\mathrm{t}} / C_{0}$ to study adsorption of dye 1 on NPs surface.

\section{Results and discussion}

\subsection{XRD analysis}

For XRD analysis, fine powder sample of ZnS NPs capped with 2-ME was mounted on the sample holder ensuring that the powder consist of randomly oriented crystallites. From figure 3 , it is observed that XRD peaks are very broad corresponding to the (1 111$)$, (2 20 ) and (3 111 ) planes. These peaks correspond to FCC structure. It is to be noted that, the peaks observed in XRD patterns match well with those of $\beta$-ZnS (cubic) reported in the ICDD powder diffraction file no. 80-0020. Intensities of the three most important peaks of ZnS, viz. $\left\langle\begin{array}{lll}1 & 1 & 1\end{array}\right\rangle,\left\langle\begin{array}{lll}2 & 2 & 0\end{array}\right\rangle$ and $\langle 3 \quad 11\rangle$ reflections corresponding to $28.65,47.78$ and $56.52^{\circ}$, respectively do not deviate from the powder diffraction file intensities. Broadening of XRD peaks indicate the formation of ZnS nanocrystals. Crystallite size of

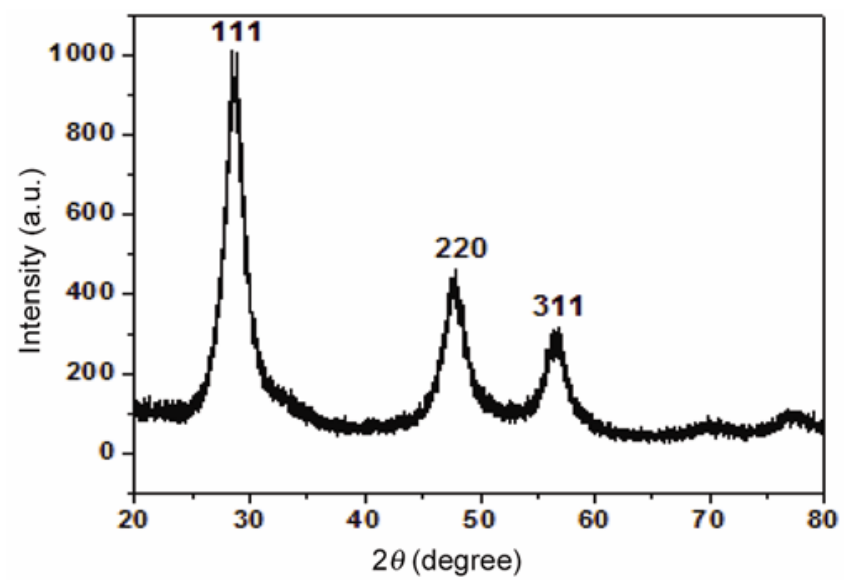

Figure 3. XRD spectra of 2-ME capped ZnS NPs.

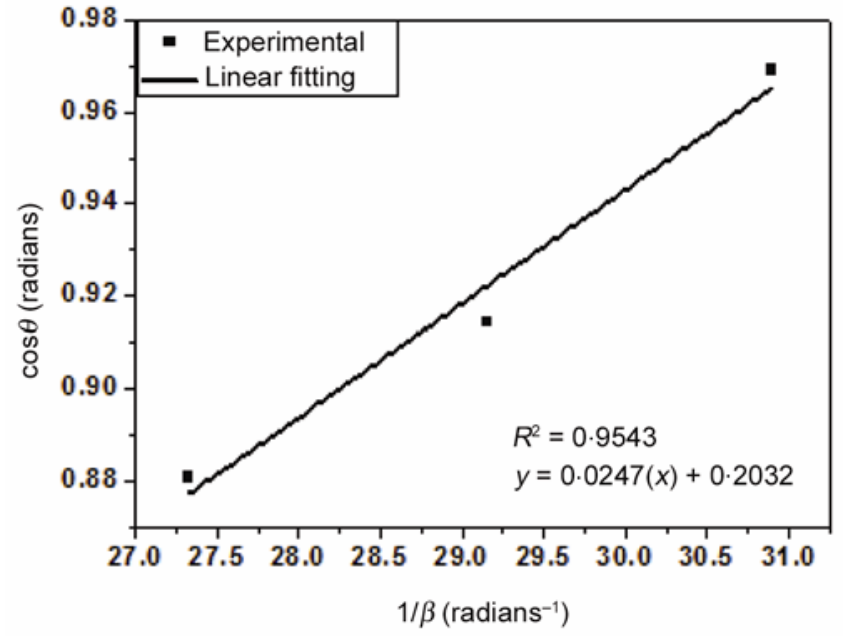

Figure 4. Scherrer's plot for ZnS. Crystallite size $(t)$ is extracted from slope of fit. 

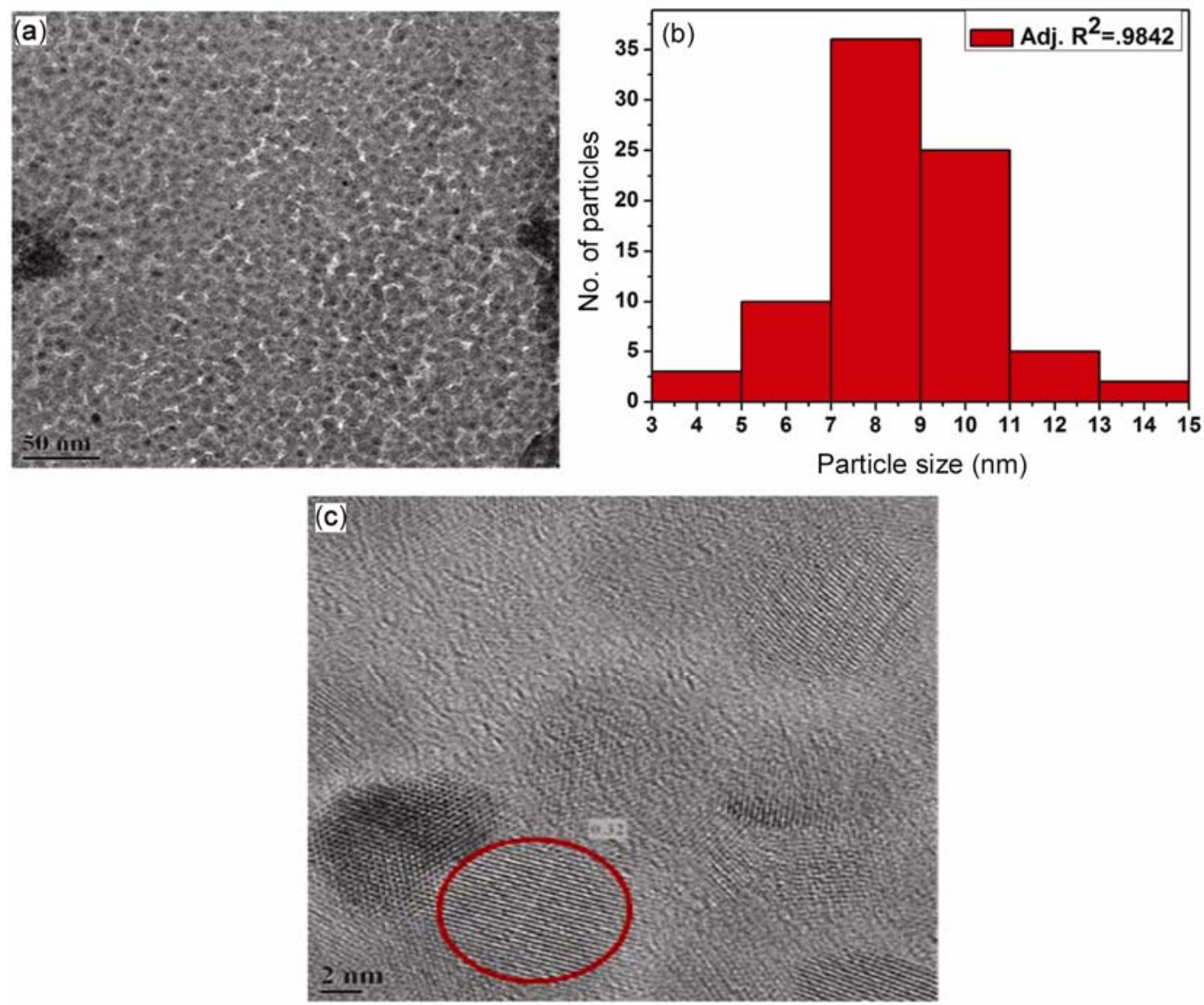

Figure 5. (a) TEM image, (b) particle size distribution and (c) HRTEM image of 2-ME capped ZnS NPs.

ZnS NPs were calculated by following the Scherrer's equation:

$$
t=\frac{k \lambda}{\beta \cos \theta} \rightarrow \cos \theta=\frac{k \lambda}{\beta t},
$$

where $k=0 \cdot 9, t$ the crystallite size $(\AA), \lambda(\AA)$ the wavelength of $\operatorname{CuK} \alpha$ radiation and $\beta$ the corrected half width of the diffraction peak (Zachariasen 1945). Plots were drawn with $(1 / \beta)$ on the $x$-axis and $\cos \theta$ along the $y$-axis for the prepared ZnS FCC phase that appeared between $2 \theta=20$ and $80^{\circ}$ (figure 4). By fitting the data linearly, the crystallite size $t$ was extracted from the slope of the fit line and it came out to be $5.6 \mathrm{~nm}$.

\subsection{TEM studies}

Figure 5(a) shows TEM micrograph of capped ZnS NPs. As can be seen in figure 5(a), ZnS NPs are spherical in shape and are nearly monodispersed. Figure 5(b) shows particle size distribution of ZnS NPs. Particle size came out to be $8.6 \mathrm{~nm}$ by lognormal fitting. It may be noted that particle size calculated from this study is larger as com- pared to XRD as the latter gives only the size of a crystallite whereas TEM gives the actual size of a NP. Due to large specific surface area and high surface energy, some nanoparticles form aggregates resulting in larger particle size. Figure 5(c) shows high resolution TEM (HRTEM) micrograph of NPs. In this micrograph, lattice fringing is clearly observed with $d$ spacing of $0.32 \mathrm{~nm}$ which corresponds to the (1 111 ) lattice plane spacing of $\mathrm{ZnS}$ in cubic phase. It indicates that NPs exhibit crystalline nature.

\subsection{DLS studies}

Hydrodynamic size and size distribution of 2-ME capped ZnS NPs were determined by DLS technique. As can be seen in figure 6 , most of the NPs $(>80)$ are in the range of 15-35 nm. Hydrodynamic size for capped ZnS NPs came out to be $31.83 \mathrm{~nm}$. It is important to mention here that DLS technique gives the size of NPs including the chain length of capping agent. In this work, the solution of NPs to be analysed was taken without washing which is done to remove excess polymer. So, in this case presence of excess polymer on the surface of NPs may be the reason for their larger size. Hence, we can say that the 
actual size of ZnS NPs is $<31.83 \mathrm{~nm}$. Polydispersity from this study came out to be $0 \cdot 18$. This means that the as-prepared ZnS NPs are nearly monodisperse which is also confirmed by TEM studies (figure 5a).

\subsection{UV-Vis analysis}

UV-Vis absorption spectra of the as-prepared ZnS NPs is shown in figure 7 . The absorption edge for capped $\mathrm{ZnS}$ NPs is observed at around $291.78 \mathrm{~nm}$ which is blue shifted as compared to its bulk counterpart (340 nm). The bandgap value was found to increase $(4 \cdot 25 \mathrm{eV})$ as compared to its bulk value $(3.54 \mathrm{eV})$ due to reduced particle size showing strong quantum confinement effect.

\subsection{FT-IR studies}

Figure 8 represents FT-IR spectra of 2-ME capped ZnS NPs recorded in the range of $4000-400 \mathrm{~cm}^{-1}$. As shown in

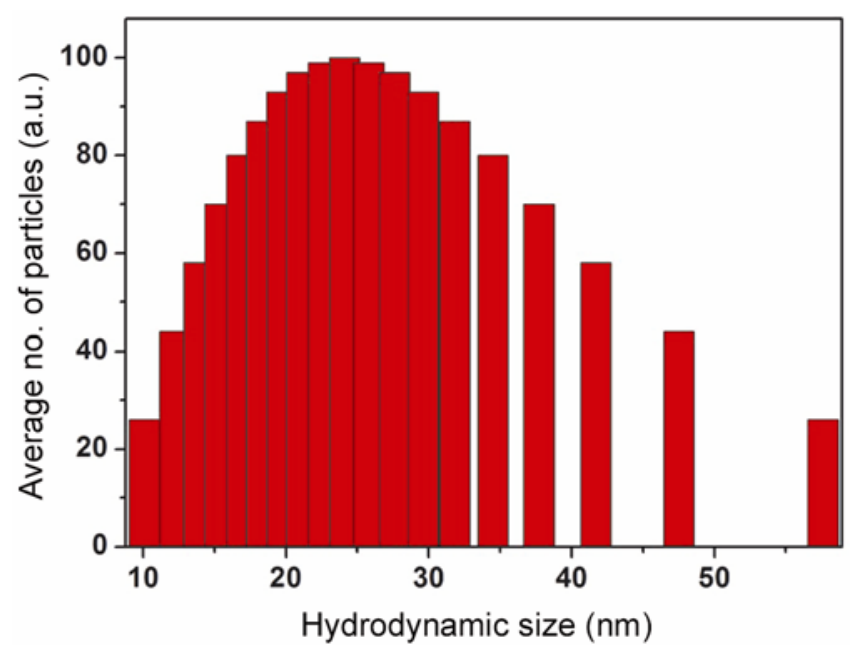

Figure 6. Histogram showing particle size distribution of capped ZnS NPs.

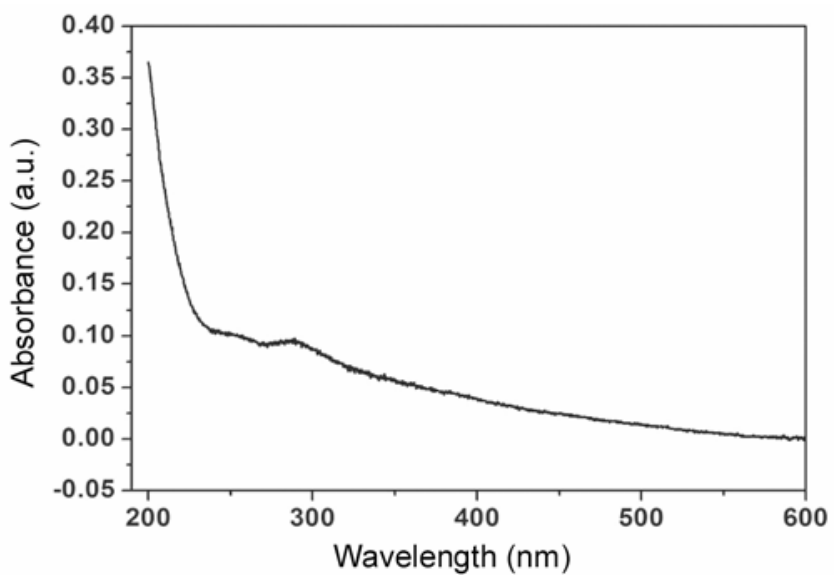

Figure 7. UV-Vis absorption spectra of 2-ME capped ZnS NPs. figure $8, \mathrm{O}-\mathrm{H}$ stretching is observed at $3305 \mathrm{~cm}^{-1}$ which is confirmed by its bending vibration occurring at $1624 \mathrm{~cm}^{-1}$ (Goswami and Sen 2004). Peaks observed at 2924 and $2874 \mathrm{~cm}^{-1}$ correspond to $\mathrm{C}-\mathrm{H}$ asymmetrical stretching vibration and symmetrical stretching vibrations, respectively. As discussed in our previous publication (Sharma et al 2010), S-H vibration observed at $2555 \mathrm{~cm}^{-1}$ in the pure 2-ME polymer was not observed for 2-ME capped $\mathrm{ZnS}$ sample. Here also, this peak was not observed. This gives a clear indication that ME was de-protonated and coordinated to the surface of ZnS NPs through sulphur (Hullavarada and Hullavarad 2008). Passivation is confirmed by the formation of S-S bond $\left(400-600 \mathrm{~cm}^{-1}\right)$, which occurs due to breaking of S-H bonds. S-S bond arises between sulphur from thiol group existing in the capping agent and sulphur in ZnS NPs. The peak observed at $1412 \mathrm{~cm}^{-1}$ can be assigned to $-\mathrm{CH}_{2}-\mathrm{O}$ for ME-capped ZnS NPs which exists from 1470 to $1300 \mathrm{~cm}^{-1}$ as reported for 2-ME polymer (Hullavarada and Hullavarad 2008). The sharp peaks observed at 1282, 1047 and $1001 \mathrm{~cm}^{-1}$ correspond to $\mathrm{C}-\mathrm{O}$ stretching of 2-ME. The sharp peak observed at $1047 \mathrm{~cm}^{-1}$ for ME-capped ZnS NPs corresponds to asymmetric stretching vibrational mode indicating a surface modification due to the passivation of $\mathrm{ZnS}$. This phenomenon proves the fact that 2-ME got assembled successfully on ZnS surface.

\subsection{Dye degradation studies}

As given in table 1, till now various dyes have been degraded using different catalysts. Photodegradation parameters like $\mathrm{pH}$ of dye solution, concentration of catalyst and reaction rate have been studied in this context. Although a vast work has been done on photocatalytic properties of different nanoparticle systems, but $\mathrm{pH}$ and reusability studies of these photocatalysts still need more

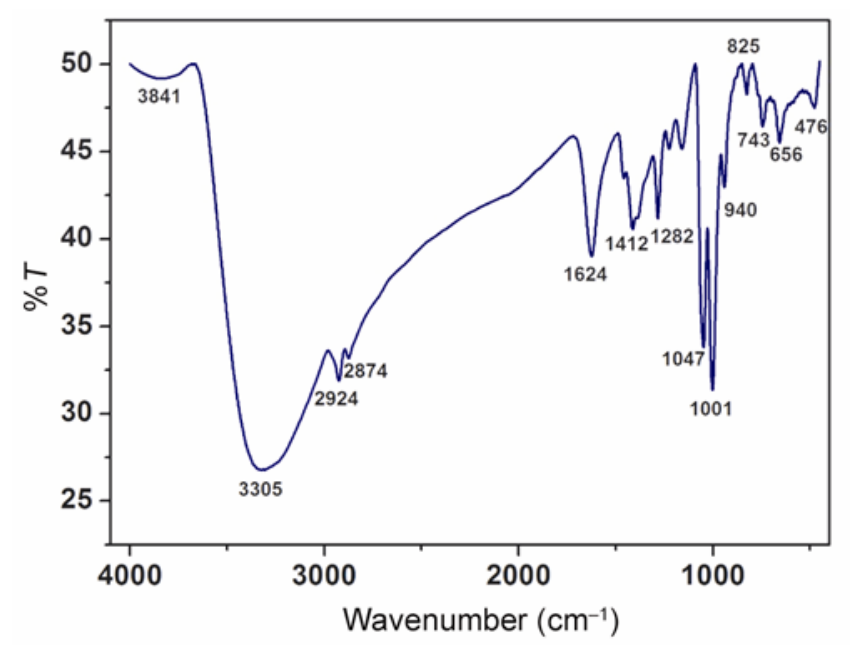

Figure 8. FT-IR spectra of 2-ME capped ZnS NPs. 


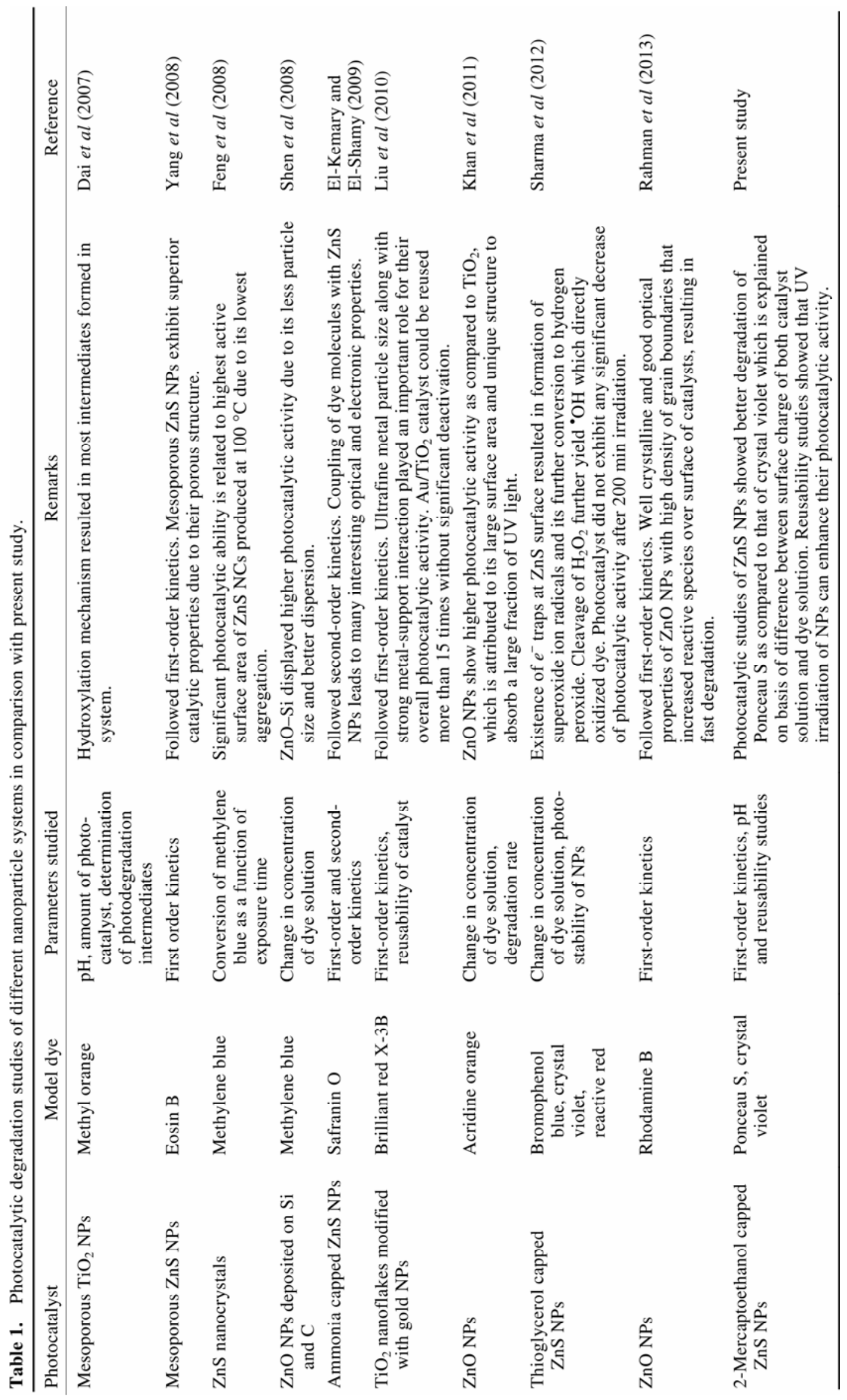


exploration. In this work, we have made an attempt to study the effect of difference in $\mathrm{pH}$ of catalyst solution and dye solution along with reusability of ZnS NPs.

Photocatalytic studies of capped ZnS NPs were done using an anionic dye (dye 1 ) and a cationic dye (dye 2). Figure $9(\mathrm{a}, \mathrm{b})$ shows degradation of dyes 1 and 2 $(10 \mathrm{mg} / \mathrm{L})$, respectively under UV-irradiation in time interval of 60 min using as-prepared catalyst $(2 \cdot 5 \mathrm{~g} / \mathrm{L})$. Degradation of dye is indicated by decrease in absorbance of samples at $\lambda_{\max }(519 \cdot 19 \mathrm{~nm}$ in case of dye 1 and $572.41 \mathrm{~nm}$ in case of dye 2) at different time intervals. Therefore, by Beer-Lambert law, the decrease in concentration of dye is recorded at different intervals of time to measure degradation rate. As can be seen in figure 9(a), when capped ZnS NPs were employed as a photocatalyst, an appreciable decrease in concentration of dye solution (dye 1) takes place. But in case of dye 2, a very less
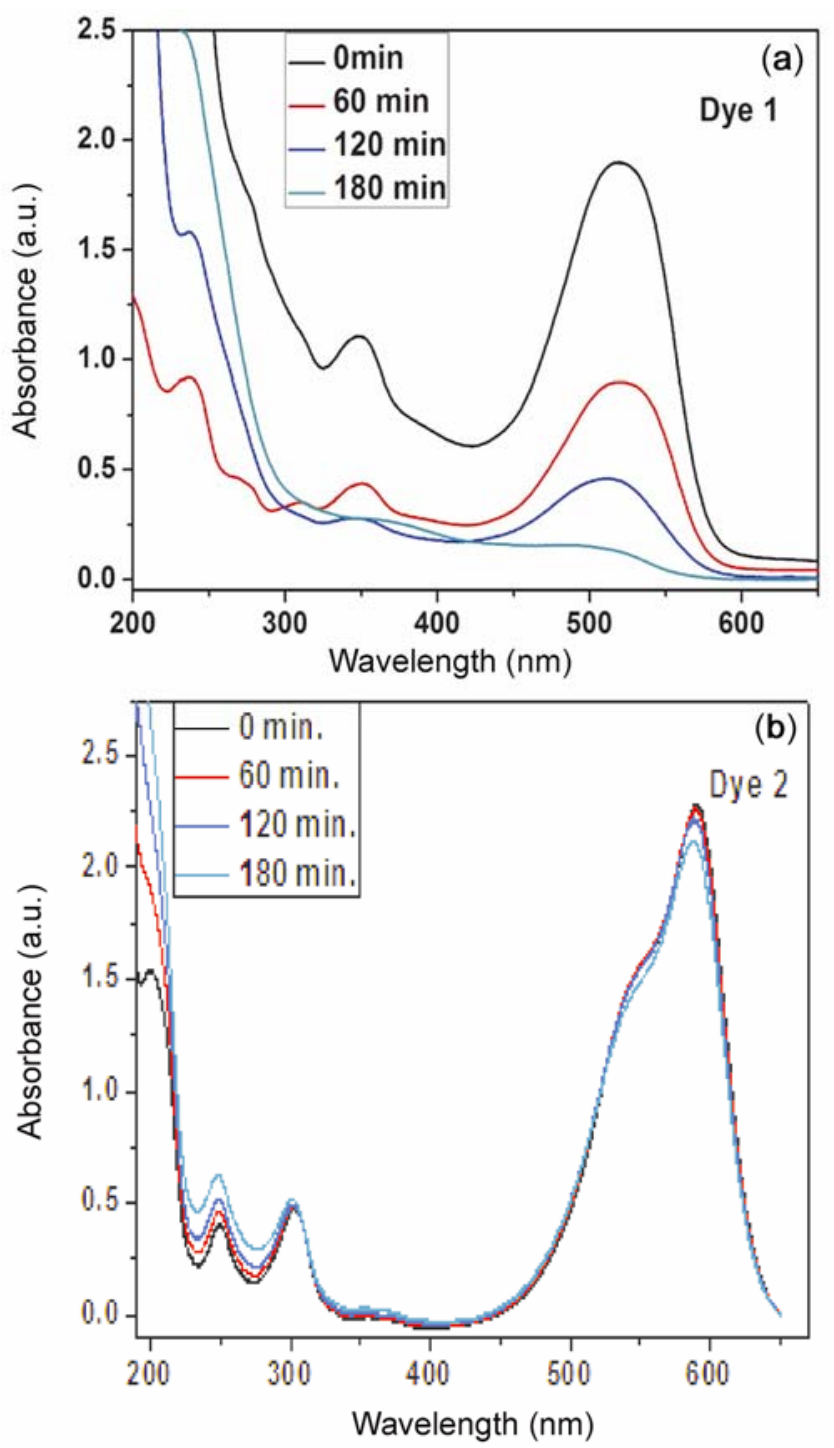

Figure 9. Absorbance spectra of (a) dye 1 and (b) dye 2 catalysed with capped ZnS NPs. decrease in concentration is observed (figure 9b). Comparison of photodegradation of both dyes $(10 \mathrm{mg} / \mathrm{L})$ catalysed with $2.5 \mathrm{~g} / \mathrm{L} \mathrm{ZnS} \mathrm{NPs} \mathrm{in} \mathrm{terms} \mathrm{of} \mathrm{change} \mathrm{in}$ concentration with respect to the initial concentration is shown in figure 10(a). The value of rate constant $(k)$ for both photochemical reactions was obtained from the slope of plots of figure 10(b). Since, in this study, concentration of solute is very low $(10 \mathrm{mg} / \mathrm{L})$, so LangmuirHinshelwood kinetics model (Lizama et al 2002) can be simplified to pseudo first order kinetic model equation:

$$
\ln \left(\frac{c_{\mathrm{t}}}{c_{0}}\right)=k T,
$$

where $C_{\mathrm{t}}$ is the concentration of dye after irradiation in selected time interval, $C_{0}$ the initial concentration of dye, $k$ the first-order rate constant and $T$ the irradiation time. By linear fitting of slopes of plots of figure 10(b), the value of $k$ in case of dye 1 came out to be $0.0146 \mathrm{~s}^{-1}$ which is higher as compared to that of dye $2\left(0 \cdot 0011 \mathrm{~s}^{-1}\right)$. Figure 11 shows percentage decrease in concentration of
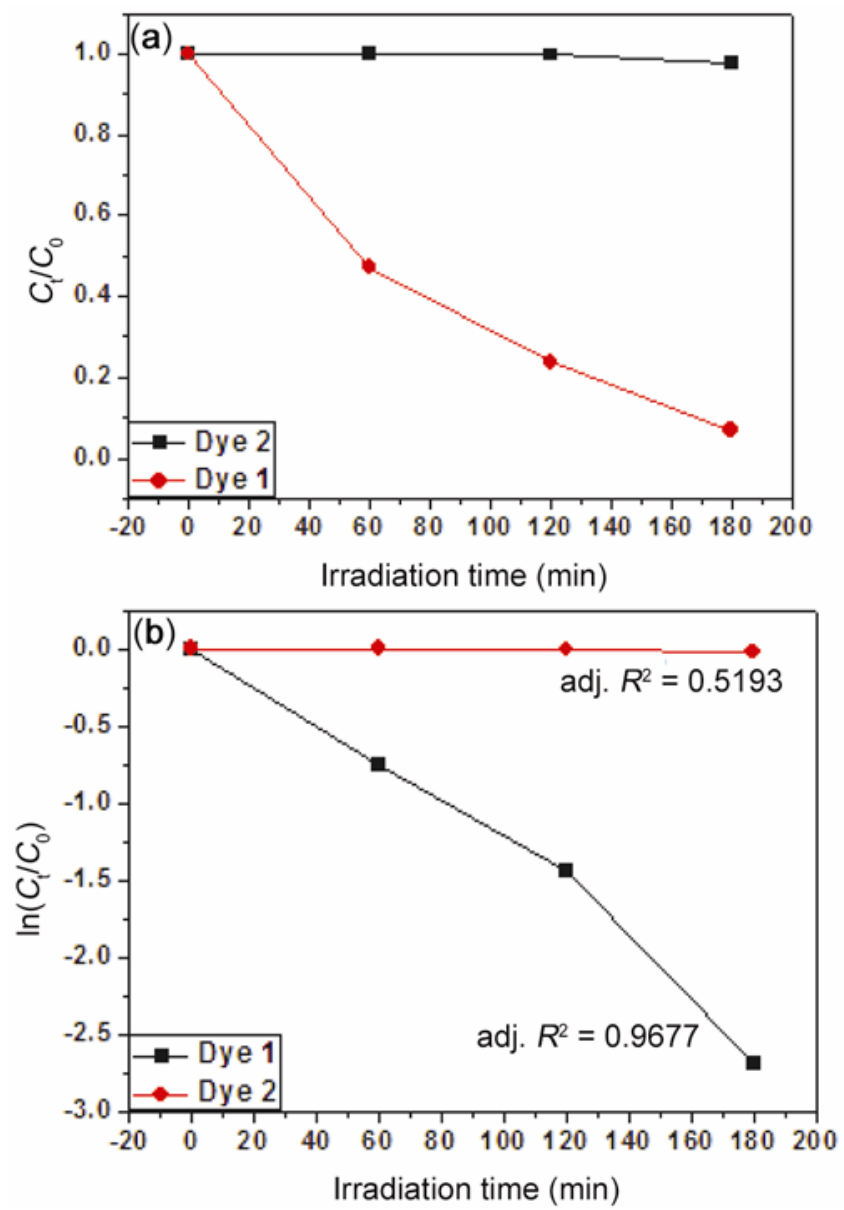

Figure 10. (a) Variation of $C_{\mathrm{t}} / C_{0}$ with irradiation time for capped ZnS NPs and (b) plot between $\ln \left(C_{\mathrm{t}} / C_{0}\right)$ and irradiation time to determine value of $k$ (rate constant). 
both dye solutions in different intervals of irradiation time. The degradation efficiency has been calculated as:

$$
\% D=\left(1-\frac{c_{\mathrm{t}}}{c_{0}}\right) \times 100,
$$

where $C_{\mathrm{t}}$ is the concentration of dye after irradiation in selected time interval, $C_{0}$ the initial concentration of dye. As shown in figure $11, \% D$ in case of degradation of dye 1 is $>(92 \cdot 6 \%)$ of dye $2(2 \cdot 3 \%)$.

As discussed earlier, $\mathrm{ZnS}$ is a semiconductor material and therefore, photodegradation of a dye is initiated by photoexcitation of semiconductor. Electron-hole pair is obtained from photoexcitation of $\mathrm{ZnS}$. The electrons combine with atmospheric oxygen to produce superoxide anions $\left({ }^{\circ} \mathrm{O}_{2}^{-}\right)$and the holes combine with hydroxide ions to produce hydroxyl radicals $\left({ }^{\circ} \mathrm{OH}\right)$. The latter defines the indirect oxidation of dyes. These free radicals are known to be very strong oxidizing agents (Gouvea et al 2000). In

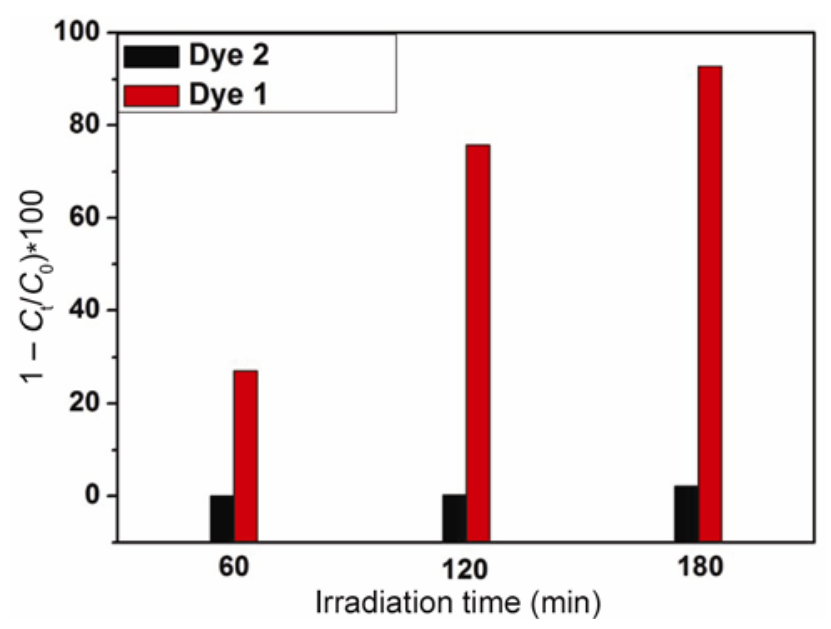

Figure 11. Degradation percentage of dyes 1 and 2 using capped ZnS NPs at different irradiation times.

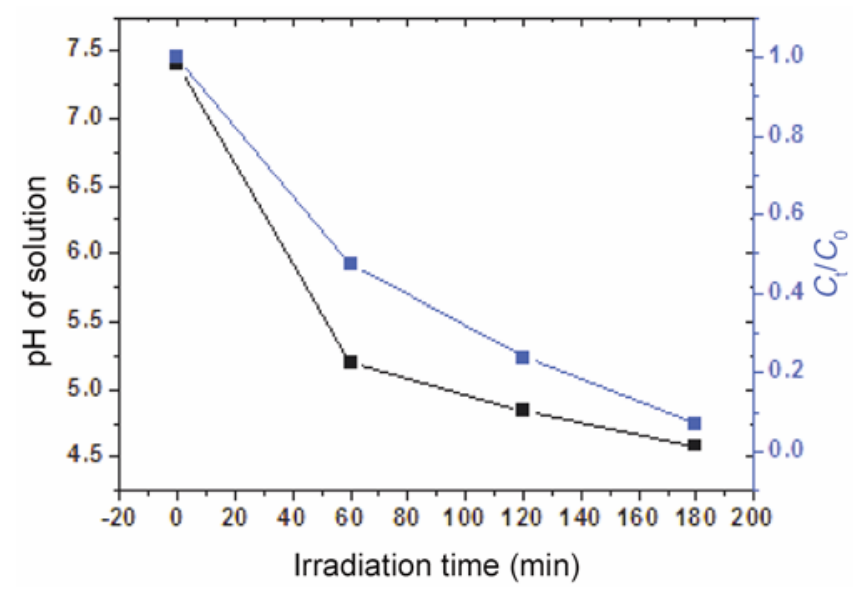

Figure 12. Variation of $\mathrm{pH}$ of dye solution (dye 1) and $C_{\mathrm{t}} / C_{0}$ with irradiation time. photocatalytic process, there are various factors which determine the photocatalytic efficiency of catalyst. Degradation efficiency depends largely on the total number of free charge carriers available on the catalyst surface. At nanoscale, the transportation length of electron-hole from crystal interface to the surface is short, which helps to accelerate the migration rate of charge carriers to the surface and hence to participate in photocatalytic process (Beydoun et al 1999). Surface charge of nanoparticles and adsorbant in aqueous solution also plays an important role in degrading the dye. Interaction of dye molecules with catalyst surface largely depends on the surface charge of both. If the dye is cationic in nature, then the catalyst surface should be negatively charged in order to have sufficient adsorption. In the present case, $\mathrm{pH}$ of dye 2 solution came out to be around 5 which is same as that of catalyst solution. But observed $\mathrm{pH}$ of dye 1 is $7 \cdot 40$. Also, as given in literature, dye 2 is cationic in nature whereas dye 1 is anionic in nature (Gupta et al 2006; Murray et al 2006). As there is comparatively large difference between the surface charge of dye 1 solution and $\mathrm{ZnS}$ NPs solution, hence, dye molecules were able to adsorb sufficiently on the surface of capped ZnS NPs. But, this is not possible in case of photocatalytic degradation of dye 2 because there is very small difference between the surface charges of both.

As described above, dye (dye 1) is degrading sufficiently with increase in irradiation time. So, there should be effect on $\mathrm{pH}$ of dye solution also. To investigate this effect, $\mathrm{pH}$ of dye 1 solution was monitored after regular intervals of time $(60 \mathrm{~min})$. It was observed that $\mathrm{pH}$ decreased with increase in irradiation time (figure 12). Also, as shown in figure $12, \mathrm{pH}$ decreased in the same manner as the concentration of dye decreased in the solution. Hence, we can say that with increasing irradiation time, there is change in surface charge of dye (or final products formed) i.e. its behaviour is transforming from anionic to cationic nature. The values of $\mathrm{pH}$ and concentration of dye 1 solution with increase in irradiation time are summarized in table 2.

\subsection{Reusability studies}

To check the reusability of catalyst, ZnS NPs were extracted after 1st use, then washed twice and were dried

Table 2. Variation in $\mathrm{pH}$ and concentration of dye 1 solution with increase in irradiation time.

\begin{tabular}{ccc}
\hline $\begin{array}{l}\text { Irradiation time } \\
(\text { min) }\end{array}$ & $\begin{array}{c}\mathrm{pH} \text { of solution } \\
\text { (dye 1) }\end{array}$ & $C_{\mathrm{t}} / C_{0}$ \\
\hline 0 & $7 \cdot 40$ & 1 \\
60 & $5 \cdot 20$ & $0 \cdot 4725$ \\
120 & $4 \cdot 84$ & $0 \cdot 2381$ \\
180 & $4 \cdot 58$ & $0 \cdot 0678$ \\
\hline
\end{tabular}




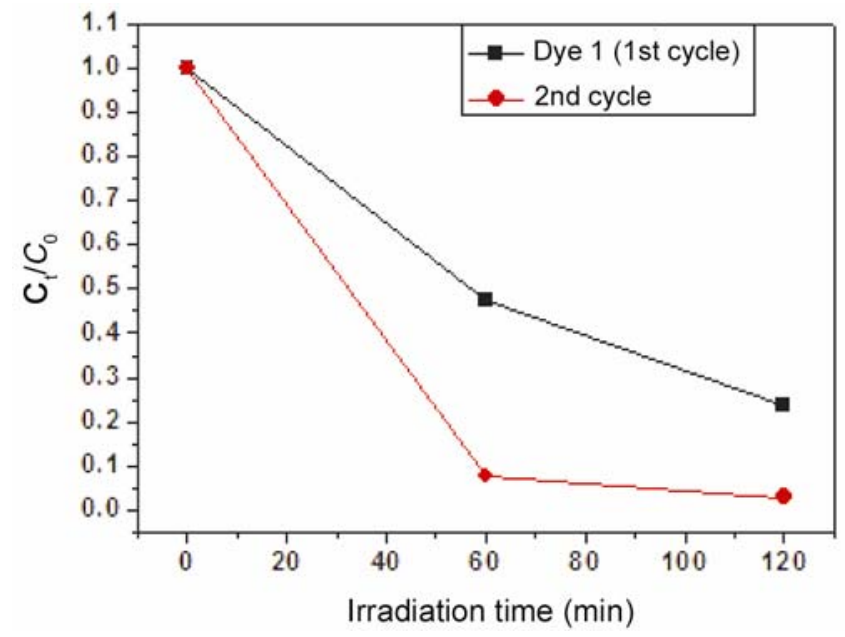

Figure 13. Variation of concentration of dye (dye 1) at different intervals of irradiatioin time using capped ZnS NPs (a) initial sample and $(\mathbf{b})$ reused sample.

at $80^{\circ} \mathrm{C}$ for $10 \mathrm{~h}$. The dried powder was again employed to use as a catalyst to degrade the same dye (dye 1). It is important to mention here that ratio of catalyst and dye was same as that used in 1st cycle. It was observed that catalyst in 2nd cycle was able to degrade the dye more rapidly as compared to that of 1 st cycle. As shown in figure 13, decrease in concentration of dye for 2nd cycle is much more than that of 1 st cycle. This unique result can be attributed to the phenomenon of UV curing that resulted in the better passivation of surface states and decrease in non-radiative recombination centres (Gallagher et al 1995). As discussed in our recent publication (Kaur et al 2013), when capped NPs are UV irradiated, then there occurs a series of chemical reactions which result in the surface modification of NPs. The surface of NP is being passivated as a result of these chemical reactions, therefore, there is lesser probability for the electronhole recombination (passivation results in decrease in probability of non radiative centres). As a result, more electrons and holes will participate in degradation of dye and hence, dye will degrade to more extent with UV irradiated catalyst as compared to that catalyst which was not UV irradiated.

\section{Conclusions}

Very fine ZnS NPs of crystallite size 5.6 nm were synthesized via chemical precipitation method. TEM study also confirmed the nanosize formation, monodispersity and high crystallinity of ZnS. From DLS results, hydrodynamic radius came out to be $31.83 \mathrm{~nm}$. Nanosize formation was also confirmed by blue shift of absorption edge as compared to the bulk counterpart of ZnS. FT-IR studies of as-prepared ZnS NPs confirmed that the capping agent
(2-ME) successfully got assembled on ZnS surface. Photocatalytic studies of capped ZnS NPs showed better degradation of dye 1 as compared to that of dye 2. The observations of this investigation clearly demonstrate the importance of choosing the degradation parameter (difference between surface charge of both catalyst solution and dye solution in this case). This parameter can be optimized to obtain a high degradation rate, which is essential for any practical application of photocatalytic processes. Also, from the reusability studies, it is concluded that UV irradiation of NPs can enhance their photocatalytic activity and hence can better degrade organic pollutants.

\section{Acknowledgement}

Authors are thankful to DRDO for funding this work through grant no. ERIP/ER/0703659/M/01/1287 dated 03-02-2011.

\section{References}

Banat M, Nigam P, Singh D and Marchant R 1996 Bioresour. Technol. 58217

Bannur V S, Kulgod V S, Metkar S S, Mahajan K S and Sainis K J 1999 Anal. Biochem. 267382

Beydoun D, Amal R, Low G and McEvoy S 1999 J. Nanopart. Res. 1439

Chung K T 1983 Mutat. Res. 114269

Chung K T, Stevens S E J and Cerniglia C E 1992 Crit. Rev. Microbiol. 18175

Dai K, Chen H, Peng T, Ke D and Yi H 2007 Chemosphere 69 1361

Drinkwater P 1990 Australian New Zealand J. Obstet. Gynecol. 3065

El-Desoky H S, Ghoneim M M and Zidan N M 2010 Desalination 264143

El-Kemary M and El-Shamy H 2009 J. Photochem. Photobiol. A: Chem. 205151

Feng S, Zhao J and Zhu Z 2008 Mater. Sci. Eng. B150 116

Gallagher D, Heady W E, Racz J M and Bhargava R N 1995 J. Mater. Res. 10870

Goswami N and Sen P 2004 Solid State Commun. 132791

Gouvea C A K, Wypych F, Moraes S G, Duran N, Nagata N and Zamor P P 2000 Chemosphere 40433

Gupta A K, Pal A and Sahoo C 2006 Dyes Pigments 69 224

Hoffmann M R, Martin S T, Choi W and Bahnemann D W 1995 Chem. Rev. 9569

Hullavarada N and Hullavarad S S 2008 J. Vac. Sci. Technol. A 261050

Jiang D, Cao L, Liu W, Su G, Qu H, Sun Y and Dong B 2009 Nanoscale Res. Lett. 478

Kanemoto M, Shiragami T, Pac C J and Yanagida S 1992 J. Phys. Chem. 963521

Kaur J, Sharma M and Pandey O P 2013 J. Nanosci. Nanotechnol. 134861 
Khan S B, Faisal M, Rahman M M and Jamal A 2011 Talanta 85943

Liu Y, Chen L, Hu J, Li J and Richards R 2010 J. Phys. Chem. C114 1641

Lizama C, Freer J, Baeza J and Mansila H 2002 Catal. Today 76235

Li X, Huan C, Liu H, Xu J, Wan B and Wang X 2011 Physica E 431071

Meena R C, Pachwarya R B, Meena V K and Arya S 2009 Am. J. Environ. Sci. 5444

Murray R F, Harper H W, Granner D K, Mayes P A and Rodwell V W 2006 Study of dye in textile industry, lange medical books (New York: McGraw-Hill)

Olad A, Behboudi S and Entezami A A 2012 Bull. Mater. Sci. 35801

Rahman Q I, Ahmad M, Misra S K and Lohani M 2013 Mater. Lett. 91170
Robinson T, McMullan G, Marchant R and Nigam P 2001 Bioresour. Technol. 77247

Sarkar D, Mukherjee S and Chattopadhyay K K 2013 Physica E 5037

Sharma M, Kumar S and Pandey O P 2010 J. Nanopart. Res. 12 2655

Sharma M, Jain T, Singh S and Pandey O P 2012 Sol. Energ. 86 626

Shen W, Li Z, Wang H, Liu Y, Guo Q and Zhang Y 2008 J. Hazard. Mater. 152172

Yanagida S, Mizumoto K and Pac C J 1986 J. Am. Chem. Soc. 108647

Yang J, Peng J J, Zou R, Peng F, Wang H, Yu H and Lee J Y 2008 Nanotechnology 19255603

Zachariasen W H 1945 Theory of X-ray diffraction in crystals (New York: John Wiley \& Sons Inc) Vol. 225 